\title{
Our panel of experts highlight the most important research articles across the spectrum of topics relevant to the field of CNS oncology
}

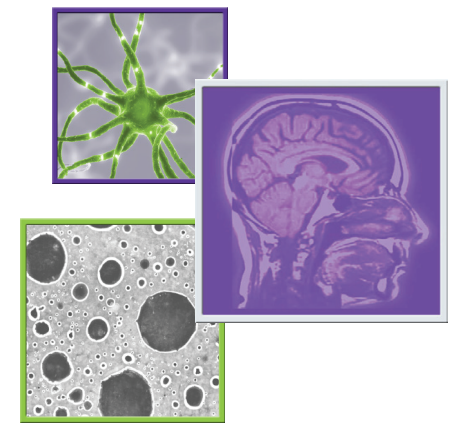

Expert panel: Larry Junck, University of Michigan Health System, MI, USA; Maura Massimino, Fondazione Istituto di Ricovero \& Cura a Carattere Scientifico Istituto Nazionale dei Tumori, Milan, Italy

Venkatesh HS, Johung TB, Caretti V et al. Neuronal activity promotes glioma growth through neuroligin-3 secretion. Cell 161, 1-14 (2015).

In this study from the laboratory of Dr Michelle Monje, Thy1::ChR2 mice bearing the excitatory opsin channelrhodopsin-2 (ChR2) and also having an implanted pediatric glioblastoma demonstrated increased tumor cell proliferation after 1 week of intermittent excitatory stimulation, manifested by $42 \%$ increase in tumor cell burden and increased indices of cell proliferation. After light stimulation of cortical slices from Thyl::ChR2 mice, conditioned media from these slices applied to cell cultures promoted proliferation index and viable cell number of pediatric and adult glioblastoma, diffuse intrinsic pontine glioma and anaplastic oligodendroglioma cells. Mass spectroscopy identified neuroligin-3, a synaptic protein, as the mediator of much of the cell proliferation, confirmed by direct application to the same glioma cell cultures. Neuroligin-3 induced PI3K-mTOR pathway activity and feedforward expression of neuroligin-3 in glioma cells. In 429 glioblastoma patients in the TCGA database, neuroligin-3 mRNA expression was found to correlate with survival (20.8 months with below-median expression, 15.2 months with above-median expression). The authors conclude that neuronal activity promotes highgrade glioma proliferation, that neuroligin-3 is a mitogen mediating this effect and that neuroligin-3 expression correlates with human glioblastoma survival.

Written by Larry Junck
Speirs CK, Simpson JR, Robinson CG et al. Impact of $1 p / 19 q$ codeletion and histology on outcomes of anaplastic gliomas treated with radiation therapy and temozolomide. Int. J. Radiat. Oncol. Biol. Phys. 91(2), 268-276 (2015).

This is a retrospective review of 111 patients with supratentorial anaplastic oligodendroglioma $(\mathrm{AO})$, oligoastrocytoma $(\mathrm{AOA})$ and astrocytoma (AA), treated by institutional practice with radiation (RT, median, 60 Gy) and concomitant temozolomide (TMZ), with or without adjuvant TMZ. Codeleted AO/AOA had superior 5-year survival $(91 \%)$ compared with non-codeleted $\mathrm{AO} / \mathrm{AOA}(68 \%, \mathrm{p}=0.02)$, whereas AA had inferior 5-year survival (37\%) compared with noncodeleted AO/AOA ( $p=0.007)$. On multivariate analysis, age, performance status and histologic/ molecular subtype were independent predictors of survival and progression-free survival. Compared with historical control data from large randomized trials, RT + TMZ provided comparable survival to $\mathrm{RT}$ with procarbazine, lomustine and vincristine for codeleted $\mathrm{AO} / \mathrm{AOA}$, superior survival to RT alone for non-codeleted AO/AOA and similar survival to RT alone for AA. These retrospective data provide preliminary evidence that adding TMZ to RT may be beneficial for patients with codeleted and non-codeleted AO/AOA but not for those with AA.

Written by Larry Junck

Kros JM, Karin Huizer K, HernándezLaín A et al. Evidence-based diagnostic algorithm for glioma: analysis of the results of pathology panel review and

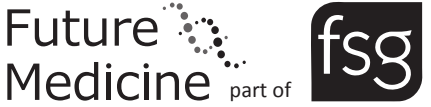


molecular parameters of EORTC 26951

and 26882 trials. J. Clin. Oncol. doi:10.1200/ JCO.2014.59.0166 (2015) (Epub ahead of print).

In this pathological tour de force, six expert neuropathologists reviewed the pathology of 636 patients enrolled in EORTC 26882 (418 patients, initially open to patients with high-grade glioma, later restricted to anaplastic astrocytoma) and EORTC 26951 (anaplastic oligodendroglial tumors). Seven molecular features were available for some patients. The data were analyzed using a conditional inference tree analysis. Large and systematic differences were seen between the pathologists; for example, individual pathologists diagnosed $38.1-63.5 \%$ of cases as glioblastoma and $4.1-31.9 \%$ as anaplastic oligodendroglioma. The diagnosis of glioblastoma by the local pathologist was confirmed by consensus (at least four of six experts) in $88 \%$. However, for anaplastic glioma subtypes, the local pathologist's diagnosis was confirmed in only $6-25 \%$, and no panel consensus could be reached in $41-51 \%$ of cases. In the inference tree analysis of combined histologic and molecular data, the three features that best separated the cases, in order of prognostic significance, were 1p/19q loss, EGFR amplification and astrocytic morphology. Surprisingly, 1p/19q loss was identified in $9 \%$ of consensus glioblastomas. This study highlights the challenges in histologic diagnosis, especially of anaplastic gliomas.

\section{Written by Larry Junck}

\section{Pajtler KW, Witt H, Sill M et al. Molecular classification of ependymal tumors across all CNS compartments, histopathological grades, and age group. Cancer Cell 27(5), 728-743 (2015)}

This paper reports on 500 worldwide collected ependymal tumors, reclassified, after pathological re-review, with DNA methylation profiling. The authors conclude by describing nine molecular subgroups including also adult subtypes (mostly subependymomas grade 1 with some rare case of ependymoma grade 2, found in supratentorial, posterior fossa and spinal comparts), almost completely absent in childhood. Of main interest for the pediatric neuro-oncologic community is the confirmed report on the existence of two posterior fossa ependymoma subgroups named PF-EPN-A, partly consistent with younger children anaplastic ependymoma, and PF-EPN-B, mostly represented by tumor affecting adolescents with both grade 2 and 3 histologic subtypes, respectively correlated with a severe and very good prognosis. Same prognostic distinction is also found in the two molecular subtypes found in supratentorial sites, the ST-EPN-RELA one, generated by a kind of chromotripsis of chromosome 11 and with an awful prognosis, and the ST-EPN-YAP1 one, correlated to copy number aberration again on chromosome 11, giving a good prognosis. They also distinguished, apart from subpendymomas as above described, two other molecularly distinguished spinal ependymoma subgroups corresponding to mixopapillary ependymoma and ependymoma with NF2 gene mutation. Putting in a statistical model also clinical characteristics with their findings, they concluded in a multivariate analysis that maintained the prognostic significance of their subtypes and confirmed the impact of complete resection and 1q gain, as the majority of other paper on ependymoma biology and outcome. They conclude with the superiority of this methylation profile on any other ependymoma classification but, of course, this will have to be demonstrated in a homogeneously treated cohort of patients like the one prospectively accrued in the recently opened SIOP (International Society of Pediatric Oncology) protocol throughout European countries. Thereafter it could be possible also to evaluate druggable targets correlated to epigenetic modifiers.

Written by Maura Massimino

DeWire M, Fouladi M, Turner DC et al. An open-label, two-stage, Phase II study of bevacizumab and lapatinib in children with recurrent or refractory ependymoma: a collaborative ependymoma research network study (CERN). J. Neurooncol. 123(1), 85-91 (2015).

This paper reports on the disappointing results of a open-label Phase II study on pediatric recurrent ependymomas. Drugs were chosen because Lapatinib, a selective ERBB1 and ERBB2 inhibitor, had produced prolonged disease stabilization in patients with ependymoma in a Phase I study and bevacizumab exposure in ependymoma xenografts leaded to ablation of tumor self-renewing cells, arresting growth. Moreover, bevacizumab in adult patients with recurrent ependymoma had demonstrated to be very efficacious [1]. Of the 20 patients evaluable for response, nobody had an objective response. The authors explained this 
lack of efficacy with the high number of previous therapy lines done by the patients and with results, coming after their study beginning, showing lack of intratumoral target inhibition by lapatinib in a study on pre-resection use of this drug in ependymoma [2]. Another one, moreover, demonstrated the lack of efficacy of bevacizumab as single agent in this patient population [GLAde Bender JL (2008)]. It is clear that target therapies need to be checked in vivo before declaring their possible success and that despite the upcoming elegant papers on biology discovery any preclinical model needs to be verified in vivo.

Written by Maura Massimino

Wright KD, Sabin ND, Cheuk D et al. Incidental diagnosis of diffuse intrinsic pontine glioma in children. Pediatr. Blood Cancer 62(6), 1081-1083 (2015).

\section{References}

1 Green RM, Cloughesy TF, Stupp R et al. Bevacizumab for recurrent ependymoma. Neurology 73, 1677-1680 (2009).

2 Fouladi M, Stewart CF, Blaney SM et al. A molecular biology and Phase II trial of lapatinib in children with refractory CNS malignancies: a pediatric brain tumor consortium study. J. Neurooncol. 11, 173-179 (2013).
Of 146 patients with pontine glioma accrued at St. Jude's Children Hospital in a 11-year time span, three were incidentally diagnosed having diagnostic MRI for other purposes than checking for neurological suspicious symptoms. This 'early' diagnoses in a devastating disease leading to death in a median of 9 months corresponded to three long-term survivors (over 2 years) with $1 / 3$ being without progression at 30 months after incidental diagnosis. The authors could also biologically analyze one of the cases finding out wild-type $\mathrm{H} 3 \mathrm{~F} 3 \mathrm{~A}$, that is the absence of the typical mutation of pontine glioma leading to death in short time. Deriving a message from this short experience, they do suppose that most long-term survivors with diffuse intrinsic pontine glioma can have a similar H3F3A status and this is an interesting hypothesis.

Written by Maura Massimino 